\title{
STATISTICALLY BASED SURVIVAL RATE ESTIMATION IN PATIENTS WITH SOFT TISSUE TUMORS
}

doi: $10.2478 /$ rojost-2018-0018

\author{
B. Şerban ${ }^{1}$, Z. Panti1,2, M. Nica ${ }^{1,2}$, M. Pleniceanu ${ }^{1}$, M. Popa ${ }^{1,2}$, R. Ene ${ }^{1,2}$, C. Cîrstoiu ${ }^{1,2}$ \\ ${ }^{1}$ Orthopedics and Traumatology Department, University Emergency Hospital, Bucharest, Romania \\ 2"Carol Davila" University of Medicine and Pharmacy, Bucharest, Romania
}

Introduction. Although most soft tissue tumors are benign, with a high healing rate after surgical excision, there is a variety of malignant tumors with differences in progression and prognosis. The study aims to assess the survival rate in patients diagnosed with this pathology, based on the patient's characteristics (age, gender, race), as well as the tumor's (histological type, differentiation degree, location and size).

Materials and methods. The retrospective study included a group of 103 patients diagnosed in our department during 2010 and 2017. Considering the high healing rate of benign tumors, only the group of neoplastic patients ( 45 cases) was involved in the survival rate estimation, assessing tumor characteristics and individual comorbidities.

Within this lot, we emphasized a predominance of neoplasm in patients aged over 50 years ( 32 cases), men (29 cases), and localization of the neoplasm in the thigh (23 cases). The predominant histopathological type, liposarcoma, was diagnosed in $67 \%$ of the cases, with dimensions over 6 $\mathrm{cm}$ and with local extension.

Results. There have been significant variations in mortality between the different histological subtypes (liposarcoma vs. synovial sarcoma). Local recurrences were shown in 18 cases of liposarcoma in the first 2 years after the surgical excision, with an increased aggressiveness of this neoplasm in men over 50 years. 12 cases developed distant metastasis, and, until the end of the study, 7 deaths were reported in 3 cases involving associated comorbidities.

Conclusions. The five-year survival is inversely proportional to the extent of the tumor and the local invasion, as well as to the age of the patient. It is difficult to appreciate an overall survival rate in the context of a heterogeneous group of tumors so it must be evaluated for every histological subtype taking into account the patient's particularities.

Keywords: survival, sarcoma, mortality, prognosis 